\title{
nature
}

genetics

\section{Donning our fancy coats}

A lmost six years have elapsed since the publication of the draft sequences of the human genome (Nature 409, 860921; 2001 and Science 291, 1304-1351; 2001). With the near-finished human genome sequence now available and draft sequences for mouse, rat, chicken, puffer fish and chimpanzee completed, efforts to annotate and characterize the functional elements of vertebrate genomes are in full force. At their heart, such efforts are grounded in the expectation that this ever-expanding knowledge of genome structure will yield fresh insights into the underlying biology of vertebrates and pave the way for a deeper understanding of human health and improved strategies for diagnosing, preventing and treating disease.

The mouse, prized for decades as a genetic model par excellence, continues to figure prominently in the current era of genome-scale biology. Partly by virtue of its historically privileged position as the model organism of choice among vertebrate geneticists, the mouse has been at the forefront of more than two decades' worth of efforts to develop tools and strategies that enable precise engineering of DNA sequence alterations that can be passed stably to offspring and analyzed genetically at the level of the whole organism. More recently, advances in genomic sequence analysis and SNP discovery have provided researchers with the necessary resources to exploit the vast levels of natural sequence variation present among widely used mouse inbred strains. As researchers begin to unravel the functional elements of vertebrate genomes and the interplay between natural variation and phenotype, the venerable mouse now sits at the nexus of these two strands that extend through much of today's genetic and genomic research.

Last fall, Nature Genetics published three consortium papers that together signaled the mouse's central role in ongoing large-scale efforts to map the functional elements of vertebrate genomes and to dissect the genetic architecture of complex phenotypic traits. The first two papers outlined broad proposals by US $(36,921-924 ; 2004)$ and European $(36,925-927$; 2004) scientists to develop a collection of knockouts for every mouse gene. The third paper $(\mathbf{3 6}, 1133-1137 ; 2004)$ described a proposal to create a community resource to facilitate the analysis of complex traits in mice. Following in the spirit of these community-driven efforts, we present The User's Guide to the Mouse, a collection of five Perspectives offering strategic advice on how best to apply the tools and resources available in the mouse to improve our understanding of genome function and biological processes relevant to human health and disease.

In an introductory piece, Claire Wade and Mark Daly present a historical overview of the origins of mouse inbred strains and discuss how this history has shaped the patterns of genetic diversity seen among laboratory strains in common use today. In a companion piece, Elizabeth Fisher and colleagues discuss the origins of the most widely used outbred mice, emphasizing current uses (and misuses) of these genetically heterogeneous stocks. Francis Stewart and colleagues follow with a discussion of current issues in mouse genome engineering, outlining strategies that allow for precisely targeted genomic alterations at almost any scale while highlighting the advantages and limitations of existing tools and reagents. Looking beyond the primary nucleotide sequence, Jesse Mager and Marisa Bartolomei explore the use of the mouse as a model system for studying complex epigenetic mechanisms and their role in normal development and disease states. A final Perspective by Geraldine Guasch and Elaine Fuchs examines the use of mice in stem cell research, highlighting the essential role of mouse studies for gaining insights into the biology of stem cells and their niches and for exploring the feasibility of stem cell-based therapies.

We thank the authors for their thoughtful contributions and diligent efforts, as well as our referees for their valuable comments during the review process. We also thank The Jackson Laboratory and Cait McKeown, respectively, for contributing the photographs of laboratory mice (photographs by Stanton Short and Kimball Wade) and of fancy mice (photographs by Cait McKeown) that appear on the front and back covers of the supplement. We hope you find the pieces that follow to be a useful and insightful guide to the world of mouse genetics. 